\title{
Cobertura geográfica del sistema mexicano de salud y análisis espacial de la utilización de hospitales generales de la Secretaría de Salud en 1998
}

\author{
Juan E Hernández-Avila, M en C, ${ }^{(1)}$ Mario H Rodríguez, MC, Dr en Filosofía, ${ }^{(1)}$ \\ N orma E Rodríguez, Lic en Inf, ${ }^{(1)}$ René Santos, Ing Elec, ${ }^{(1)}$ Evangelina Morales, Ing Ind, ${ }^{(1)}$ \\ Carlos Cruz, M en $C_{,}^{(2)}$ Jaime Sepúlveda-A mor, Dr en C.(1)
}

\begin{abstract}
Hernández-Avila JE, Rodríguez MH, Rodríguez NE, Santos R, Morales E, Cruz C, Sepúlveda-Amor J. Cobertura geográfica del sistema mexicano de salud y análisis espacial de la utilización de hospitales generales de la Secretaría de Salud en 1998. Salud Publica Mex 2002;44:519-532.
\end{abstract} El texto completo en inglés de este artículo está disponible en: http://www.insp. mx/salud/index.html

\section{Resumen}

Objetivo. D eterminar la cobertura geo gráfica del Sistema Mexicano de Salud y analizar la utilización en 1998 de los hospitales de la Secretaría de Salud (SSA). Material y métodos. Se desarrolló un Sistema de Información Geográfica (SIG) con información sociodemo gráfica por localidad y ubicación espacial de unidades de atención de todo el sector salud, así como el registro de egresos por hospital de la SSA. Se determinó la utilización en 217 hospitales generales de la SSA mediante un modelo de estimación de máxima verosimilitud, que incluyó información sobre los recursos humanos, la infraestructura adicional y la población $25 \mathrm{~km}$ a la redonda. Resultados En 1998, 10806 localidades con 72 millones de habitantes contaban con al menos una unidad de atención de salud del sector público y $97.2 \%$ de la población se encontraba a menos de $50 \mathrm{~km}$ de una, pero más de 18 millones de personas vivían en localidades rurales sin unidades de atención. El promedio de ocupación fue de $48.5 \pm 28.5$ por cada 100 camas/año, con gran variabilidad intra e interestatal. La utilización se asoció significativamente con el número de los médicos en la unidad, y en unidades del Instituto Mexicano del Seguro Social con la infraestructura adicional e índice de marginación. Conclusiones La utilización del SIG eleva la capacidad analítica y proporciona estimadores más realistas de la cobertura y utilización de ho spitales del sector. El texto completo en inglés de este artículo está disponible en: http://www.insp.mx/salud/index.html

Palabras clave: sistemas de información geográfica; cobertura; utilización; servicios de salud; México

\author{
Hernández-Avila JE, Rodríguez MH, Rodríguez NE, \\ Santos R, Morales E, Cruz C, Sepúlveda-Amor J. \\ Geographical coverage of the Mexican Healthcare System \\ and a spatial analysis of utilization \\ of its General Hospitals in 1998. \\ Salud Publica Mex 2002;44:519-532. \\ The English version of this paper \\ is available at: http://www.insp.mx/salud/index.html
}

\begin{abstract}
A bstract
Objetive. To describe the geographical coverage of the Mexican Healthcare System (MHS) services and to assess the utilization of its General Hospitals. Material and Methods. A Geographic Information System (GIS) was used to include sociodemographic data by locality, the geographical location of all MHS healthcare services, and data on hospital discharge records. A maximum likelihood estimation model was developed to assess the utilization levels of 217 MHS General Hospitals. The model included data on human resources, additional infrastructure, and the population within a $25 \mathrm{~km}$ radius. Results. In 1998, 10,806 localities with 72 million inhabitants had at least one public healthcare unit, and $97.2 \%$ of the population lived within 50 $\mathrm{km}$ of a healthcare unit; however, over 18 million people lived in rural localities without a healthcare unit.The mean annual hospital occupation rate was $48.5 \pm 28.5$ per 100 bed/years, with high variability within and between states. Hospital occupation was significantly associated with the number of physicians in the unit, and in the Mexican Institute of Social Security units utilization was associated with additional health infrastructure, and with the population's poverty index. Conclusions. GIS analysis allows improved estimation of the coverage and utilization of MHS hospitals.The English version of this paper is available at: http://www.insp.mx/salud/index.html
\end{abstract}

Keywords: geographic information systems; coverage; utilization; health services; Mexico

(1) Instituto Nacional de Salud Pública,A venida Universidad 655, colonia Santa María A huacatitlán, 62508 Cuernavaca, Morelos, México.

(2) Universidad Iberoamericana, Prolongación Paseo de la Reforma 880, colonia Lomas de Santa Fe, 01210 México, D.F., México.

Fecha de recibido: 8 de octubre de 2001 • Fecha de aprobado: 3 de junio de 2002

Solicitud de sobretiros: Juan E Hernández-Avila. Dirección de Informática y Geografía Médica, Instituto N acional de Salud Pública, Avenida Universidad 655, colonia Santa MaríaA huacatitlán. 62508 Cuernavaca, Morelos, México. Correo electrónico: juan_eugenio@ correo.insp.mx 
E n el sector salud mexicano existen diversos sistemas informáticos para la captación, recolección y organización de las estadísticas básicas de morbi-mortalidad, así como de la infraestructura de atención de la salud y los servicios. Estos sistemas, administrados por las diferentes instituciones que conforman el sector, funcionan de manera independiente $y$, a menudo, con claves de codificación incompatibles que ocasionan dificultades en el análisis integral de la información del sector.

Adicionalmente, los procesos de agregación estadística y organización de la información, desde su origen en las comunidades y unidades aplicativas, su paso por los niveles jurisdiccionales y estatales, hasta alcanzar el nivel central, ocasionan en muchos casos una pérdida de resolución ${ }^{1}$ que afecta la capacidad analítica integral de los datos locales. Esta situación, originada en parte por las limitaciones impuestas por la tecnología para el manejo electrónico de datos, propició la generación de sistemas piramidales de información, que han sido muy útiles para la toma de decisiones y formulación de políticas nacionales, pero que ofrecen pocas ventajas para el análisis integral, con desglose local, de la información que recolectan, lo que dificulta su utilización en procesos analíticos en el ámbito de la comunidad.

En la última década se han desarrollado herramientas informáticas que permiten conservar el nivel de resolución original de los datos, y que hacen posible la integración de la información de diversas fuentes en un sistema relacional de bases de datos. Estas herramientas han enriquecido las capacidades analíticas y, en la actualidad, es posible integrar información sobre salud e infraestructura de atención, con información demográfica, socioeconómica, del entorno fisiográfico y su asociación con coordenadas geográficas.

La aplicación de sistemas de información geográfica $^{2}$ (SIG) para el desarrollo de un sistema integral de información sobre el sector salud cubriría las necesidades informáticas para la planeación estratégica y la investigación científica. La utilización de un sistema de esta naturaleza en el análisis de cobertura, que incorpore el componente espacial en forma cuantitativa, elevaría la capacidad analítica y proporcionaría estimadores más realistas de la cobertura del sector y de las necesidades de atención. Además, con este sistema sería posible integrar, de manera simultánea en el análisis, otros componentes como la oferta interinstitucional de servicios, la demanda poblacional y aspectos sociodemográficos, para investigar el comportamiento de los niveles de utilización de la infraestructura hospitalaria a la luz de la demanda poblacional y la oferta de servicios de atención.
La utilización rutinaria de SIG permitirá el desarrollo de estrategias operativas para compartir, de manera racional y equitativa, la infraestructura tecnológica en las unidades médicas y evitar que se presente capacidad ociosa en una institución, mientras no ha sido resuelta la demanda de servicios en otra, favoreciendo así el concepto y operación de "Redes virtuales para la prestación de servicios de salud." ${ }^{3}$

Con el propósito de investigar la cobertura geográfica del sistema mexicano de salud (SMS) y el estudio de las variables relacionadas con los niveles de ocupación en hospitales generales de la Secretaría de Salud (SSA) se construyó una base de datos integral geo-referenciada sobre el SMS con la información producida en 1998 por los diferentes sistemas informáticos existentes actualmente en el sector. Aquí se presentan los principales resultados.

\section{Material y métodos}

\section{Fuentes de datos}

Se utilizaron las bases de datos de infraestructura hospitalaria de la Dirección de Informática y Estadística de la SSA (DGIESSA), de egresos hospitalarios (DGIESSA), del Sistema Unico de Información del Instituto Mexicano del Seguro Social (IMSS) (SUI1098, IMSS) $¥$ del censo de infraestructura de salud del sector privado (DGIESSA) y del Registro Nacional de Infraestructura en la Salud (RENIS98, DGIESSA), todos para el año de 1998. La información del sector salud se combinó con datos demográficos de localidades con más de tres casas habitadas, contenidos en la base de datos de integración territorial de 1995 (ITER95) $^{\S}$ del Instituto Nacional de Estadística, Geografía e Informática (INEGI), \# el Sistema para la Consulta de Información Censal SCINCE95 (INEGI) y con las bases de datos de marginación por localidad y municipio 1995, editadas por el Consejo Nacional de Población (Conapo). \& Estas bases de datos fueron clasificadas, indexadas y geo-referenciadas en el Núcleo de Acopio y Análisis de Información en Salud (NAAIS) del Instituto Nacional de Salud Pública (INSP) para construir un sistema global nacional de información geográfica sobre infraestructura del SMS.

\footnotetext{
* Dirección General de Estadística y Evaluación en Informática. Dirección General de Informática y Estadística (DGIE). Secretaría de Salud Pública, 1998

‡ Instituto Mexicano del Seguro Social, 1998.

\& Catálogo de Integración Territorial INEGI, 1995 (ITER95).

\# Instituto Nacional de Estadística, Geografía e Informática, 1995.

\& Consejo Nacional de Población.
} 


\section{Construcción de un sistema de información geográfica}

Se construyó una base de datos geo-referenciados por localidad con la información demográfica y sobre infraestructura y recursos humanos del SMS para 1998. Esta base de datos contiene los datos de 9357 unidades de atención de la SSA, 3391 del IMSS-Solidaridad, 1274 del IMSS y 2683 del sector privado.

La información fue geo-referenciada utilizando como base cartográfica la base de datos del ITER95, que contiene la ubicación espacial de las localidades del país con más de tres casas habitadas. También se utilizaron los datos de la cartografía censal municipal del conteo nacional de población de 1995, que ofrece la división política municipal del país. La base de datos RENIS98 contenía la clave INEGI de las localidades donde se encontraban las unidades aplicativas del sector público, además, para las unidades del IMSS se registró la clave presupuestal, con esta información fue posible relacionar la base geo-referenciada con el SUI10-98.

La información en cada una de las bases de datos que dio origen a este sistema geo-referenciado presentaba diferentes tipos de indicadores. Sin embargo, para su integración, los datos básicos de infraestructura (tipo de hospital), recursos materiales y humanos (camas censables y personal médico) de todas las unidades fueron combinados en forma directa para determinar la infraestructura instalada en cada localidad del país.

\section{A nálisis descriptivo de la cobertura geográfica del sector público}

Para estimar la cobertura geográfica de atención de la salud del sector público se realizó un análisis para medir la distancia lineal existente entre las localidades y las unidades de atención más cercanas. Se estimó la población, con base en las comunidades, y se clasificó de acuerdo con la distancia que existe entre su lugar de residencia y la localidad más cercana con servicios de salud. Esta estimación se llevó a cabo construyendo círculos concéntricos (buffers), a intervalos regulares $(25,50,75$ y $100 \mathrm{~km})$ alrededor de cada localidad que contaba con servicios de salud de primer o segundo nivel de atención. Para cada buffer se determinó el número de localidades y se estimó la población total. En este análisis de cobertura se incluyó información de todas las unidades del sector público y todas las localidades del país con más de tres casas habitadas.

\section{Modelo para el análisis de la utilización de hospitales generales de la SSA}

La información disponible sobre egresos hospitalarios de la SSA contenía los registros de egreso de 356 hospitales de segundo y tercer nivel de atención, centros de salud con hospitalización (híbridos) y hospitales psiquiátricos. Estos últimos, por la naturaleza de los padecimientos tratados, producen muy pocos egresos y el tiempo promedio de estancia es muy alto y, por lo tanto, fueron descartados de los procesos analíticos para el desarrollo del modelo de utilización. La información sobre egresos hospitalarios en el Distrito Federal no se encontraba disponible en la base de datos, por lo que éstos no pudieron ser incluidos en el proceso analítico. Tomando en cuenta estas consideraciones, la base de datos sólo incorporó información sobre 298 hospitales ubicados en el interior del país. Esta base de datos contenía información sobre 61 centros de salud con hospitalización (híbridos), tres clínicas de especialidades, 17 hospitales especializados y 217 hospitales generales. La construcción del modelo de utilización se llevó a cabo empleando únicamente los datos de los hospitales generales, esto con el fin de analizar unidades de tipo similar.

Para cada hospital general se incorporó el número de camas censables, el total de egresos hospitalarios de 1998 y se calculó la duración de cada registro, utilizando para ello las fechas de ingreso y egreso contenidas en la base de datos. También se calcularon el promedio de estancia y el total anual de días/paciente por hospital. La tasa anual de ocupación hospitalaria (TAOH) se calculó, para cada hospital, mediante la siguiente ecuación:

$$
\mathrm{TAOHj}=\left(\begin{array}{c}
\sum_{\mathrm{i}=1}^{\mathrm{n}_{\mathrm{j}}} d u r_{i, j} \\
\text { camas }_{j}{ }^{* 365}
\end{array}\right)
$$

en donde $j$ se refiere a cada hospital, $i$ se refiere a cada

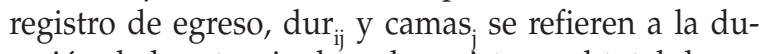
ración de la estancia de cada registro y al total de camas censables en el hospital $j$, respectivamente. El factor 365 se incorporó para incluir la disponibilidad de camas censables durante un año. $\sum d u r_{i, j}$ es el total anual de días/paciente (TADP) y $n$ es el número de registros de egreso en el hospital $j$.

Con el fin de investigar el comportamiento de la TAOH en los 217 hospitales generales de la SSA, se elaboraron gráficos de caja (box plots) para evaluar su 
variación por estado, así como gráficos de dispersión (scatter plots) para analizar su relación con el número de camas censables y el personal médico por unidad. A estos últimos se les ajustó un modelo de regresión local ${ }^{4,5}$ para suavizar la nube de puntos debido a que estas relaciones no son lineales. Los modelos de regresión local son una alternativa atractiva para el análisis de datos no lineales, debido a que la forma de la curva ajustada depende sólo de los datos, y que se basa en el ajuste de curvas de tal forma que cada punto en ellas tiene como base la información de ese punto y la de un conjunto de puntos vecinos especificados de antemano.

El análisis espacial de la TAOH inició con la construcción de un círculo (buffer) con un radio arbitrario de $25 \mathrm{~km}$ alrededor de cada hospital general (figura 1). Dentro de cada buffer, se contó el total de hospitales de la SSA, del IMSS y del sector privado, así como el número total de médicos y el total de camas censables por institución. También se contaron las localidades, la población total y se calculó el número de camas por cada 100000 habitantes. En estos modelos se incluyó, de manera simultánea, la infraestructura del IMSS y del sector privado para evaluar la ocupación hospitalaria de la SSA con el fin de corregir por la oferta de otras instituciones del sector salud (expresada por la capacidad instalada).

Con estas nuevas variables se construyó un modelo estadístico para estimar los niveles de utilización hospitalaria (expresados por la $\mathrm{TAOH}$ ) e investigar su relación con la infraestructura adicional instalada en el área de $25 \mathrm{~km}$ alrededor de cada hospital.

Como variable dependiente en este modelo se utilizó el TADP. El análisis gráfico de esta variable (discreta y positiva) determinó que su distribución se asemejaba a la de Poisson. Dadas sus características, se asumió esta distribución para la construcción del modelo estadístico y se ajustó por el número de camas disponibles durante el año (offset) en cada unidad de acuerdo con el siguiente modelo:

$$
\log \left(\mathrm{TADP}_{i}\right)=\beta_{0}+\beta_{1}+\ldots \beta_{p}+\text { offset }\left(\text { camas }^{*} 365\right)+e_{i}
$$

en donde $\mathrm{e}_{\mathrm{i}}$ se refiere al término de error, y tiene una distribución de Poisson.

Como variables predictoras, el modelo fue ajustado por la demanda poblacional (representada por el total de habitantes dentro del buffer) y la oferta adicional de servicio instalada (representada por el total de camas y médicos adicionales en clínicas y hospitales dentro del buffer). El modelo fue ajustado utilizando el método de estimación de máxima verosimilitud. ${ }^{6}$ Esta metodología es una extensión del modelo de re- gresión lineal que permite el ajuste de modelos a variables que no siguen la distribución normal, pero que pertenecen a la familia exponencial de distribuciones, como la distribución binomial o la de Poisson. La significancia de los resultados se reporta con un nivel de confiabilidad de $95 \%$.

Este modelo dio como resultado el valor esperado del TADP y con éste se calculó la TAOH esperada según el tamaño del hospital, la población y la infraestructura adicional disponible (IMSS, SSA y sector privado) en las inmediaciones de cada unidad estudiada.

La construcción del SIG y el análisis espacial de la información se hizo utilizando MapInfo (MapInfo Corporation, 2000). El análisis estadístico se llevó a cabo utilizando el paquete S-Plus (Insightful Corporation, 2000).

\section{Resultados}

La información disponible actualmente sobre infraestructura en el sector salud fue integrada exitosamente en un SIG y se logró combinarla con información sobre egresos hospitalarios de la SSA y con información demográfica y socioeconómica producida en otros sectores.

Del total de unidades geo-referenciadas, 532 de la SSA correspondieron al segundo y tercer nivel de atención: 269 hospitales generales, 27 hospitales especializados, 47 clínicas de especialidades, 25 hospitales psiquiátricos (granjas) y 164 centros de salud con hospitalización. Del IMSS fueron 346: 211 hospitales generales de zona, 104 unidades de medicina familiar y 31 hospitales de especialización.

\section{Distribución de clínicas y hospitales (cobertura geográfica)}

Utilizando las capacidades de visualización espacial del SIG se produjeron mapas de la distribución geográfica de las unidades de primer nivel de atención del IMSS-Solidaridad y de la SSA. También se determinó la ubicación de las unidades de segundo y tercer nivel de atención del sector público, y de las clínicas y hospitales del sector privado (figura 2).

En 1998, 10806 localidades (poco más de 10\% del total de localidades con tres o más casas habitadas en el país) contaban con al menos algún tipo de unidad de atención de salud del sector público. En estas localidades residían más de 72 millones de personas. Más de 18 millones de personas vivían en localidades rurales dispersas sin unidades fijas de atención. Ciento un municipios, con más de 200000 habitantes, dis- 


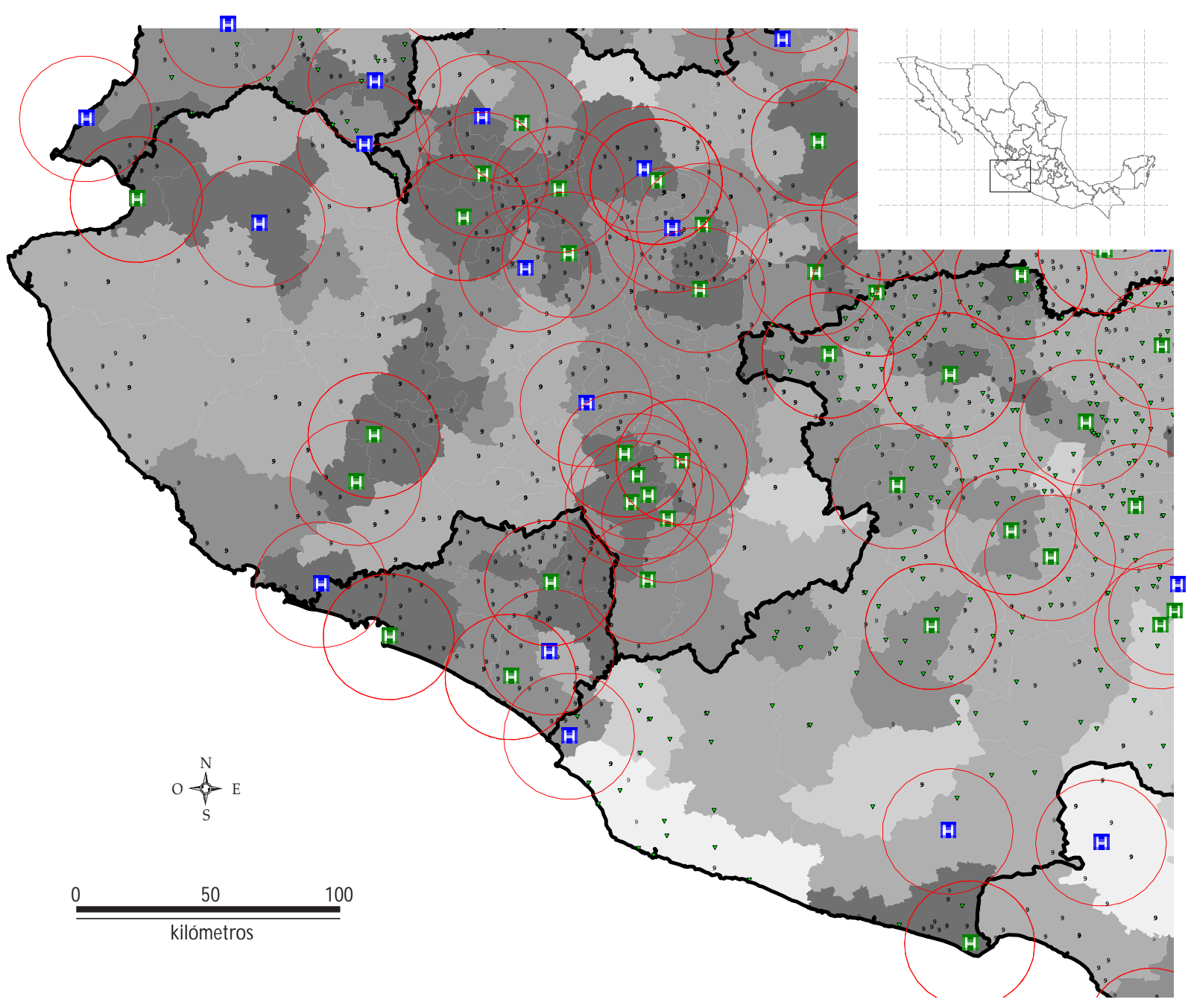

Figura 1. Detalle de la construcción de variables con referencia espacial. Se trazó una Área circular (buffer) ALREDEDOR DE CADA HOSPITAL ESTUDIADO, EN ESTA ÁREA SE IDENTIFICARON LAS LOCALIDADES Y UNIDADES DE ATENCIÓN presentes (Secretaría de Salud, Instituto Mexicano del Seguro Social y privadas). Con esta información se CALCULÓ LA POBLACIÓN TOTAL EN EL BUFFER (ÁREA DE INFLUENCIA), ASI COMO LA INFRAESTRUCTURA Y RECURSOS HUMANOS Y MATERIALES ADICIONALES A LAS DE LA UNIDAD EN ESTUDIO. CoMO SE PUEDE APRECIAR EN LA FIGURA, EXISTEN HOSPITALES QUE COMPARTEN Áreas de INFLUENCIA. LoS PUNTOS AZULES INDICAN LOCALIDADES EN LAS QUE SÓlO EXISTEN UNIDADES DE LA SSA Y LOS VERDES EN LOS QUE TAMBIÉN HABía UNIDADES DEL IMSS

tribuidos en los estados de Chiapas, Oaxaca, Puebla y Veracruz, no contaban con unidades fijas de atención de salud.

El tamaño promedio de las localidades que contaban, como mínimo, con un hospital de segundo nivel de atención fue de 22137 habitantes, sin embargo, se identificaron 100 localidades mayores de 20000 habitantes, distribuidas en 24 estados, que no contaban con servicios de este tipo (figura 3). También se observó que $89 \%$ de la población se ubicaba a distancias menores de $25 \mathrm{~km}$ de una unidad de atención de salud de segundo nivel (cuadro I) y que $97 \%$ dentro de un radio de $50 \mathrm{~km}$. Mediante el análisis espacial de la distribución geográfica de unidades de segundo nivel de atención se identificaron 1949 comunidades en el norte del país situadas a más de $100 \mathrm{Km}$. de dis- 


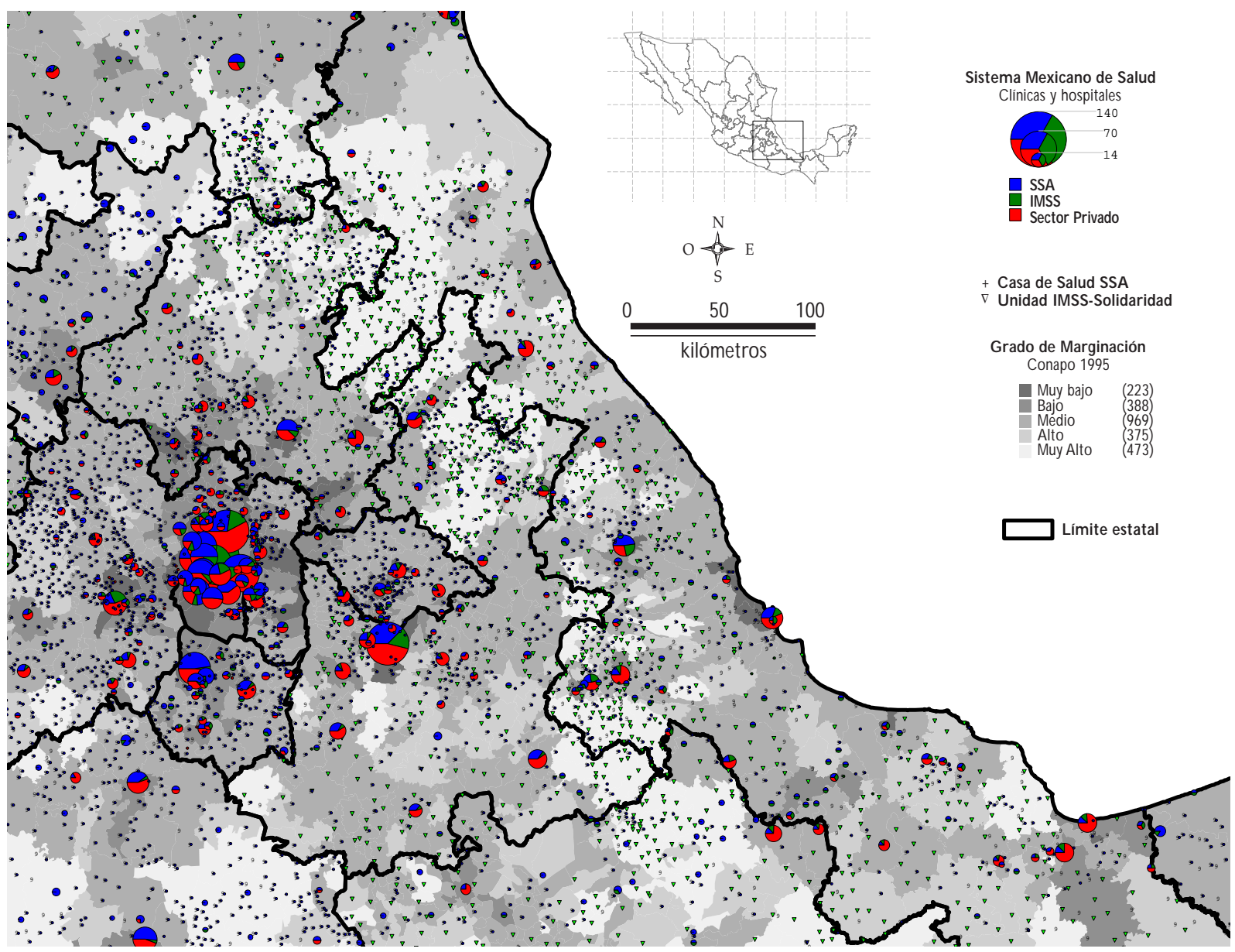

Figura 2. Distribución de las unidades de atención del sistema mexicano de salud en 1998. En esta figura SE PResenta la Región Central del país. Los Segmentos en los gráficos de pastel representan el Número TOTAL DE UNIDADES POR SECTOR PARA CADA LOCALIDAD. LOS PUNTOS REPRESENTAN LA UBICACIÓN DE UNIDADES DE PRIMer nivel de atención eXClusivamente. Sólo se presentan las localidades QUe cuentan con al menos ALGUNA UNIDAD FIJA DE ATENCIÓN DE LA SALUD. EL FONDO EN TONOS GRIS REPRESENTA EL GRADO DE MARGINACIÓN por municipio en 1995, según los datos editados por el Consejo Nacional de Población

tancia de la unidad de atención de segundo nivel más cercana (figura 4). En estas comunidades habitaban más de 180 mil personas. En el mapa se puede apreciar que las localidades en estas condiciones se situaban en regiones de alta marginación.

\section{Utilización y demanda en hospitales generales de la SSA}

$\mathrm{La} \mathrm{TAOH}$ en los hospitales estudiados presenta gran variabilidad intraestatal e interestatal (figura 5), con un promedio de $48.5 \pm 28.5$ por cada 100 camas disponi- bles durante el año. La variación de la TAOH con respecto al número de camas y personal médico presentó una pronunciada tendencia positiva cuya pendiente disminuye drásticamente después de las 30 camas y 29 médicos por unidad, respectivamente (figuras 6a y 6b). La duración media de estancia hospitalaria fue 1.76 días para atención del parto y de 4.00 días para condiciones patológicas. No se encontró asociación entre el número de médicos por cama y el promedio de la duración de la estancia hospitalaria.

En el cuadro II se presentan los resultados del modelo ajustado (coeficientes, errores estándar y signi- 
Cuadro I

Distribución de La población de aCUERdo CON LA DISTANCIA LINEAL AL HOSPITAL DE SEGUNDO NIVEL DE ATENCIÓn MÁs CERCANO. MÉXICO, 1998

\begin{tabular}{|c|c|c|c|}
\hline $\begin{array}{l}\text { Distancia a unidades } \\
\text { de segundo nivel } \\
\text { de atención }\end{array}$ & $\begin{array}{l}\text { Número de } \\
\text { localidades }\end{array}$ & $\begin{array}{l}\text { Tamaño } \\
\text { promedio de } \\
\text { la localidad }\end{array}$ & $\begin{array}{c}\text { Población } \\
\text { total }\end{array}$ \\
\hline
\end{tabular}

\begin{tabular}{lrrrr} 
Menos de $25 \mathrm{~km}$ & 62346 & 1284 & 80108584 & 88.96 \\
\hline 25 a $50 \mathrm{~km}$ & 27438 & 264 & 7254015 & 8.06 \\
\hline 50 a $75 \mathrm{Km}$ & 9179 & 220 & 2018936 & 2.24 \\
\hline 75 a $100 \mathrm{~km}$ & 3141 & 155 & 486027 & 0.54 \\
\hline 100 a $150 \mathrm{~km}$ & 1923 & 95 & 182144 & 0.20 \\
\hline Más de $150 \mathrm{~km}$ & 26 & 75 & 1940 & 0.002
\end{tabular}

ficancia). Los coeficientes se interpretan como el cambio en el logaritmo del TADP por unidad de cambio en cada una de las variables incluidas en el modelo, ajustando por el tamaño del hospital.

Los resultados del modelo (cuadro II) indican una asociación positiva con el número de médicos en la unidad de estudio $(p=0.001)$, así como con los médicos adicionales de la SSA $(p=0.039)$ y del IMSS $(p=0.008)$ en otras unidades ubicadas en la misma localidad. El modelo también señala una asociación inversa, es decir que la TADP disminuye de acuerdo con el número de hospitales de la SSA en el área de $25 \mathrm{~km}$ alrededor de la localidad en donde se encuentra el hospital $(p<0.001)$. El grado de marginación presenta una asociación inversa, estadísticamente significativa $(p<0.001)$, con la

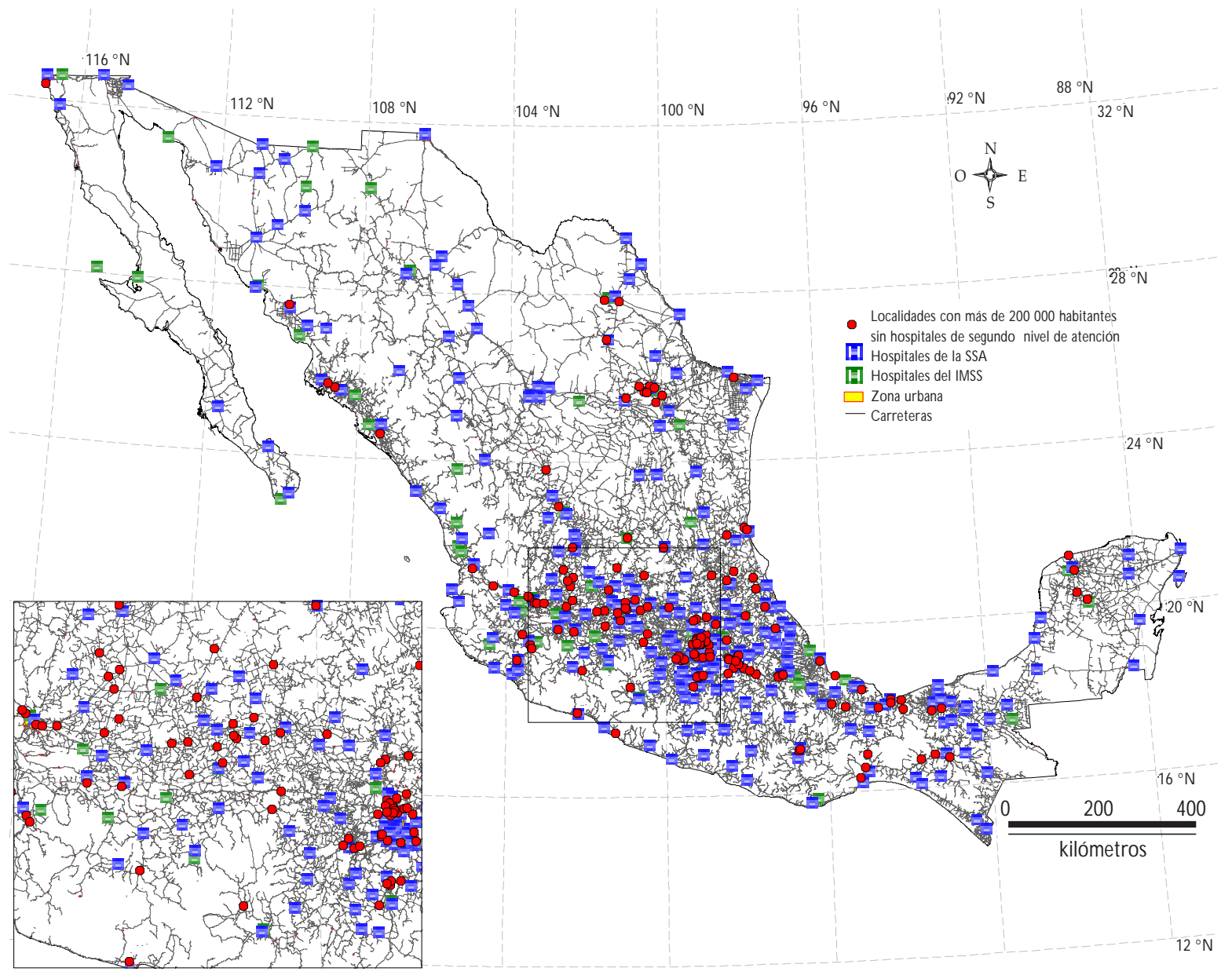

Figura 3. Distribución de localidades con más de 20000 habitantes que no contaban, en 1998, con unidades DE SEGUNDO NIVEL DE ATENCIÓN DE LA SALUD. 


\section{Resultados del modelo estadístico. México, 1998}

Variable

Coeficiente

Error estándar

Valor $\mathrm{t}$

Valor $p$

\begin{tabular}{lcccc} 
Médicos en la unidad & 0.00127 & 0.00042 & 3.02474 & 0.00140 \\
\hline Médicos adicionales en la localidad Instituto Mexicano del Seguro Social & 0.00154 & 0.00063 & 2.42429 & 0.00810 \\
\hline Médicos adicionales en la localidad Secretaría de Salud & 0.00032 & 0.00018 & 1.76533 & 0.03949 \\
\hline Total de hospitales adicionales en el área Secretaría de Salud & -0.02900 & 0.00582 & -4.98087 & 0.00000 \\
\hline Grado de marginación & -0.52300 & 0.03070 & -17.03032 & 0.00000 \\
\hline Total de clínicas privadas en la localidad & 0.00863 & 0.00389 & 2.22036 & 0.01374 \\
\hline Total de camas privadas en la localidad & -0.00081 & 0.00032 & -2.50158 & 0.00657
\end{tabular}

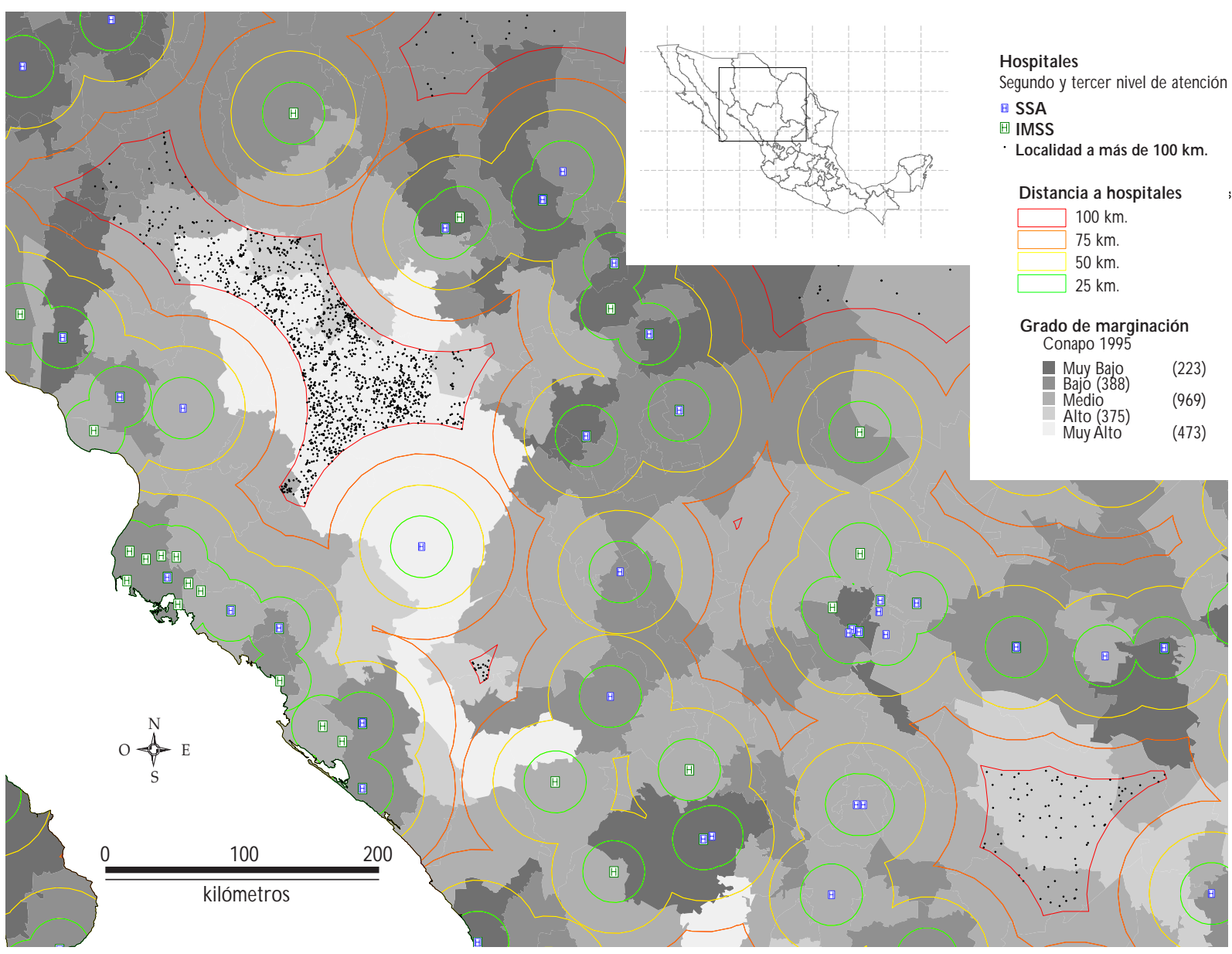

Figura 4. Distribución de las unidades de atención de segundo y tercer nivel del Instituto Mexicano del Seguro Social y la Secretaría de Salud en 1998, y localidades que se encuentran a más de 100 km de disTANCIA LINEAL DE LA UNIDAD MÁS CERCANA. ESTAS LOCALIDADES SE UBICAN EN ÁREAS RURALES DISPERSAS DE ALTO GRADO DE MARGINACIÓN 
ocupación hospitalaria, lo que sugiere mayores tasas de ocupación en localidades de baja marginación. El número de clínicas privadas en la localidad en la que se encuentra la unidad en estudio tuvo una asociación positiva con el nivel de ocupación hospitalaria ( $p=$ 0.014 ) pero el número de camas privadas presentes en la localidad se asoció con una disminución en los niveles de ocupación ( $p=0.007)$.

Los gráficos de diagnóstico del modelo (figura 7) se comportaron conforme a lo esperado, confirmando que la distribución asumida para la variable dependiente (TADP) fue la correcta. El gráfico de la figura 6a, muestra la relación entre el número de días paciente ajustados por el modelo y los observados; la nube de puntos se despliega homogéneamente alrededor de la línea recta indicando una alta correlación. El gráfico de residuales (diferencias entre lo observa- do y lo esperado de acuerdo con el modelo, figura 7b) presenta las diferencias entre el TADP observado en cada hospital y los ajustados por el modelo. En él, se indican 10 hospitales que presentaron desviaciones extremas. Por último, el gráfico de normalidad para los residuales (figura 7c) indica que éstos se comportan de acuerdo con la distribución normal, confirmando la pertinencia del modelo estadístico empleado y el apego a los supuestos del método de estimación. Los residuales del modelo fueron geo-referenciados con respecto a la unidad que les dio origen. Se observó la coexistencia de unidades con grandes diferencias en la TAOH y la existencia de áreas, como en el norte del país, donde había hospitales con residuales negativos (número de días paciente menor a lo esperado) aun cuando no había otras unidades de atención cercanas (figura 8).

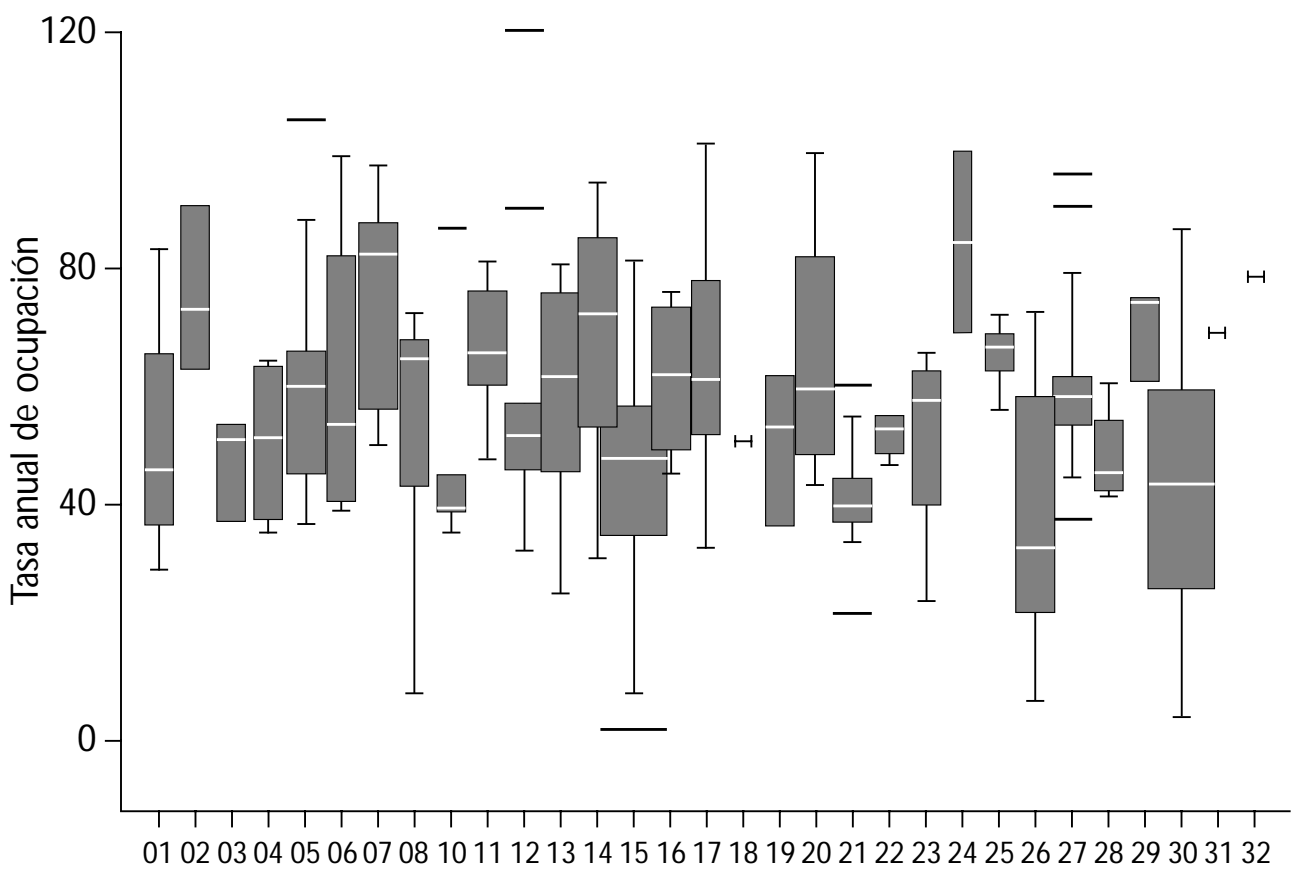

Clave de entidad federativa

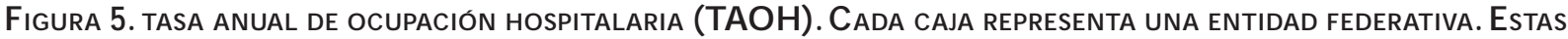
están ordenadas alfabéticamente. La entidad federativa 09: Distrito Federal no se encuentra en este gráFICO. El LARGO DE LAS CAJAS REPRESENTA UNA MEDIDA ROBUSTA DE LA VARIABILIDAD DENOMINADA RANGO INTERCUARTICULAR; EN CONJUNTO CON LAS LÍNEAS VERTICALES QUE PARTEN DE SUS EXTREMOS INDICA LA VARIABILIDAD EN LATAOH PARA CADA ENTIDAD; LA LÍNEA BLANCA EN CADA CAJA INDICA LA MEDIANA DE LA TAOH. EL ANCHO DE LA CAJA ES UN indicativo de LA CANTIDAD DE UNIDADES ESTUdiadas EN CADA ESTADO. Se PUede Ver QUe El Estado de México (15) ERA UNA DE LAS ENTIDADES CON MÁS HOSPITALES, PERO CON UNA TAOH RELATIVAMENTE BAJA, CON POCA VARIABILIDAD ENTRE HOSPITALES 
Variación del porcentaje de ocupación de acuerdo con el número de camas censables en la unidad
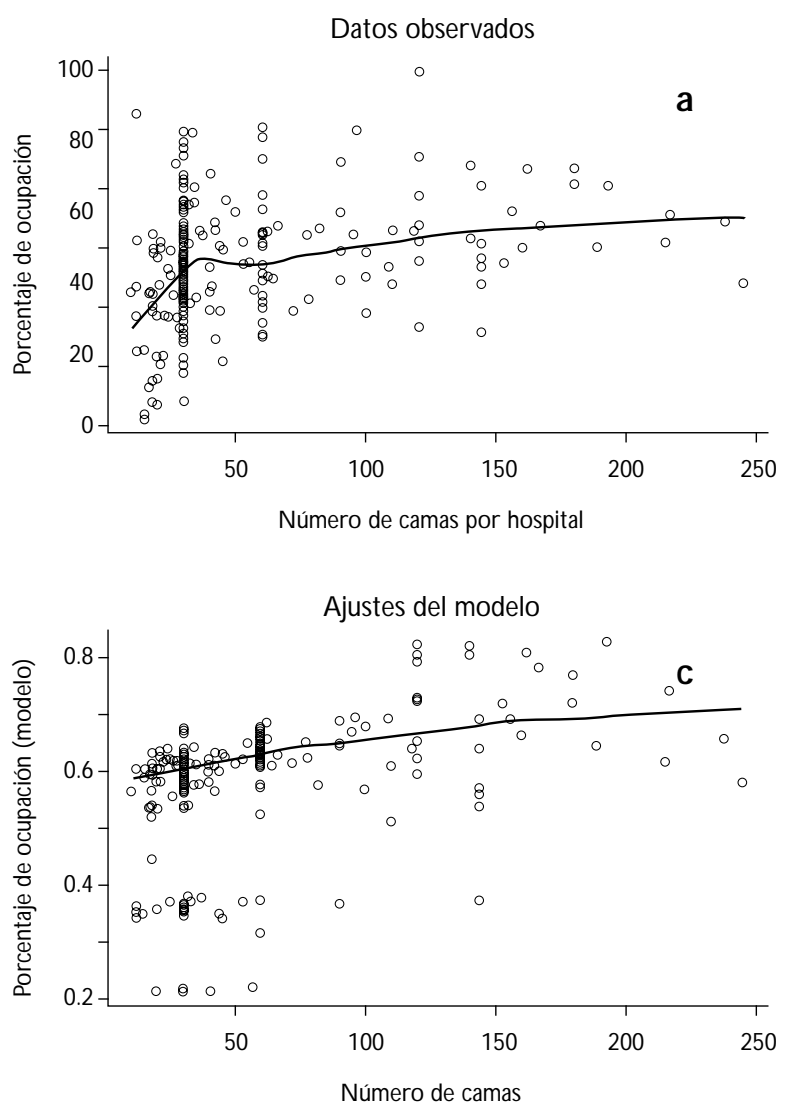

Variación del porcentaje de ocupación de acuerdo con el número de médicos en la unidad
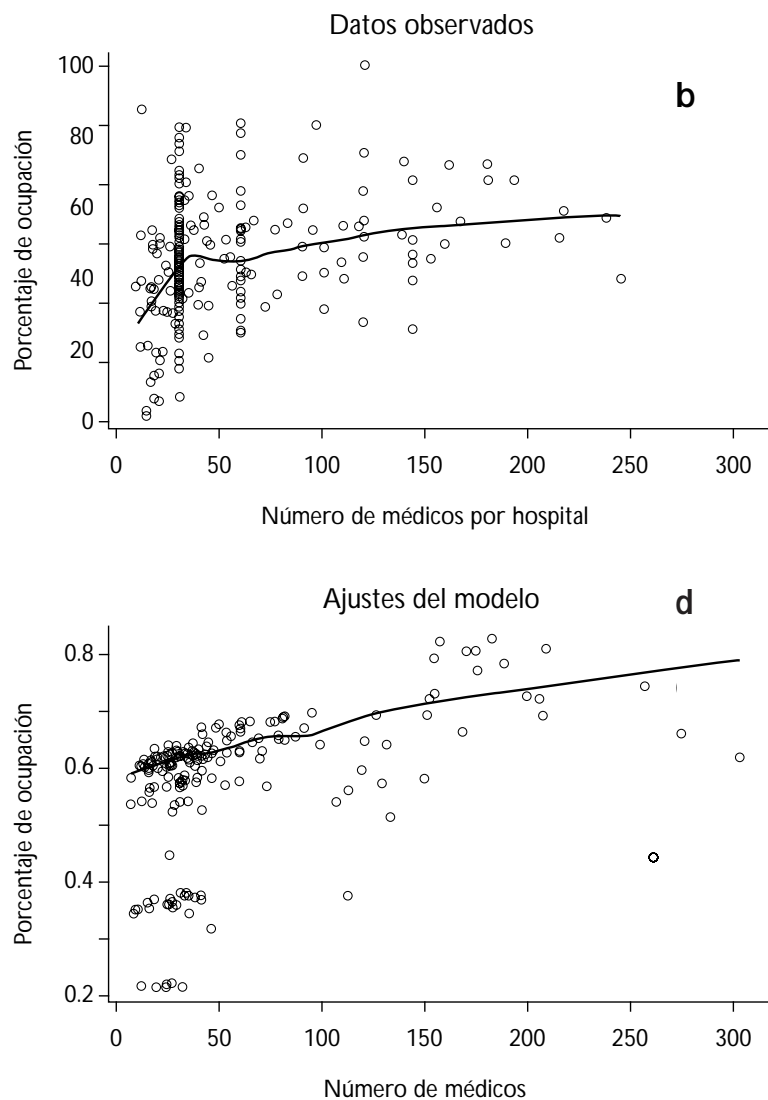

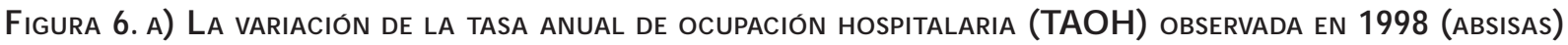
PRESENTA UNA PRONUNCIADA TENDENCIA ASCENDENTE CONFORME EL NÚMERO DE CAMAS POR HOSPITAL ASCIENDE (ordenada), sU pendiente declina a partiR de las 30 camas. B) Se observa el mismo comportamiento en la TAOH CON RESPECTO AL NÚMERO DE MÉdICOS POR HOSPITAL (ORDENADA), LA PENDIENTE DECLINA A PARTIR DE LOS 29 MÉDICOS. LAS GRÁFICAS C Y D MUESTRAN LA VARIACIÓN DE LATAOH ESTIMADA POR EL MODELO RESPECTO AL NÚMERO DE CAMAS Y MÉDICOS POR HOSPITAL, RESPECTIVAMENTE. LAS LÍNEAS CONTINUAS EN LAS GRÁFICAS REPRESENTAN EL AJUSTE DEL MODELO DE REGRESIÓN LOCAL. SE PUEDE OBSERVAR QUE LOS NIVELES ESPERADOS SON SUPERIORES A LOS OBSERVADOS. LAS NUBES DE PUNTOS SE ENCUENTRAN MÁS ESTRECHAMENTE DISTRIBUIDAS A LO LARGO DE LAS LÍNEAS. EN AMBOS GRÁFICOS SE PUEDE OBSERVAR UN CONJUNTO DE HOSPITALES PEQUEÑOS QUE OPERAN CON UNA TAOH MUY BAJA

\section{Discusión}

Nuestros resultados indican que la información disponible, producida actualmente por los sistemas informáticos con los que operan las instituciones del sector salud y de otros sectores en México, puede ser integrada en un SIG. El sistema desarrollado fue útil para la visualización de las variables de interés y tam- bién para la generación de nuevas variables basadas en su ubicación geográfica. Estas se emplearon en modelos estadísticos para la evaluación del desempeño de hospitales generales de la SSA.

Se observó que en 1998 el tamaño promedio de las localidades con al menos un hospital de segundo nivel de atención fue de alrededor de 22000 habitantes; sin embargo, esta cifra debe ser re-evaluada en el 

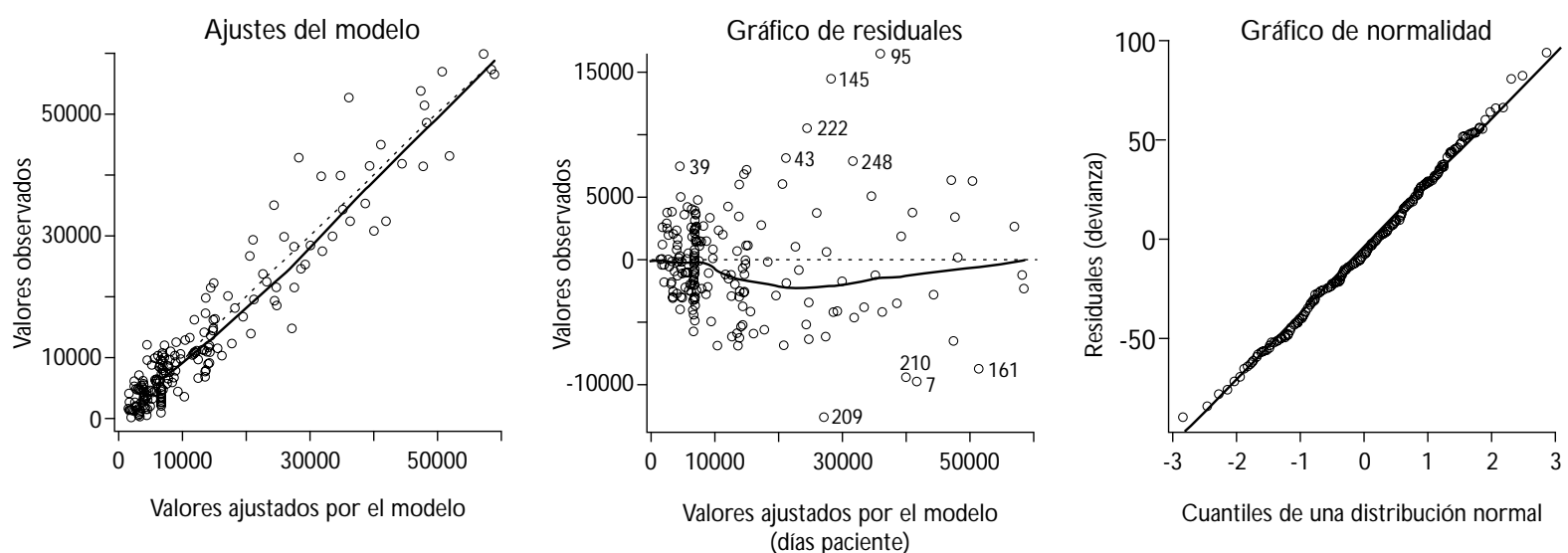

Figura 7. Gráficos de diagnóstico del modelo de regresión. El análisis gráfico de los residuales es una TÉCNICA COMÚNMENTE UTILIZADA PARA EVALUAR EL AJUSTE DE UN MODELO ESTADÍSTICO A LOS DATOS DE UN FENÓMENO OBSERVADO. EL GRÁFICO DE AJUSTE DEL MODELO INDICA QUE LOS VALORES ESPERADOS DE ACUERDO CON EL MODELO SON PARECIDOS A LOS OBSERVADOS Y QUE SE DISPERSAN ALREDEDOR DE UNA LÍNEA CON PENDIENTE CASI IGUAL A UNO. A MEDIDA QUE EL NÚMERO DE Días PACIENTE AUMENTA, LA VARIABILIDAd TAMBIÉN; ESTO SE VE MÁs CLARAMENTE EN EL GRÁFICO DE RESIDUALES, EL CUAL SE CONSTRUYÓ A PARTIR DE LA DIFERENCIA ENTRE LOS DATOS OBSERVADOS Y LOS ESTIMADOS POR EL MODELO; ESTE AUMENTO ES CONSISTENTE CON LA SUPOSICIÓN DE QUE LA VARIANZA DE UNA DISTRIBUCIÓN DE

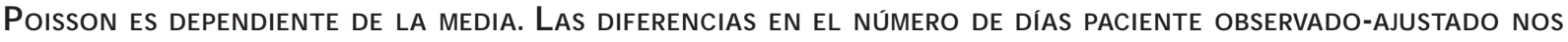
PERMITE IDENTIFICAR HOSPITALES CUYA TAOH ES MUY BAJA O ALTA CON RESPECTO A LA ESPERADA PARA UNIDADES CON LAS MISMAS CARACTERISTICAS. LA DISTRIBUCIÓN ESPERADA DE LOS RESIDUALES DEBE SEGUIR UNA DISTRIBUCIÓN NORMAL $O$ GAUSIANA, ESTO SE DEMUESTRA EN EL GRÁFICO DE NORMALIDAD EN EL QUE SE GRAFICAN LOS CUANTILES DE LA DISTRIBUCIÓN DE LOS RESIDUALES CONTRA LOS CUANTILES DE UNA MUESTRA DE IGUAL TAMAÑO PROVENIENTE DE UNA DISTRIBUCIÓN NORMAL ESTÁNDAR

marco de la red integrada de servicios de salud, para determinar el estándar de la población a cubrir por un hospital general.

Las capacidades de visualización y análisis espacial que proporciona el sistema desarrollado nos permitió realizar un análisis descriptivo sobre la cobertura del sector y la demanda poblacional. El análisis permitió ubicar a poblaciones que carecían o se encontraban lejos de las unidades de atención de la salud, pero también nos dio una idea de la dispersión de la población rural y las dificultades para llegar con los servicios de salud hasta sus comunidades, donde el aislamiento y su reducido tamaño dificultan la instalación de servicios permanentes de atención. Estos resultados indican que uno de los retos en la atención de la salud, para 1998, era el fortalecimiento en la prestación de servicios a la población rural. Esto fue atendido mediante el despliegue de unidades móviles, pero se requiere diseñar nuevos modelos de atención que garanticen la cobertura integral, de forma permanente y anticipatoria al daño en esta población. La aplicación de SIG en la planeación estratégica y operación de los recursos móviles podría optimizar su aprovechamiento y mejorar los niveles de cobertura y la oportunidad en la prestación de este tipo de servicios. El análisis presentado indica que en 1998 en los estados de Chiapas, Oaxaca, Puebla y Yucatán había deficiencias que hacían patente la necesidad de desarrollar programas especiales para superar la falta de servicios fijos de salud y que garantizaran la cobertura permanente en varios de los municipios de estos estados. En un futuro los sistemas de información geo-referenciados podrán ser empleados también para evaluar la cobertura factible de las unidades de medicina familiar y de los centros de salud, con el fin de garantizar que todo mexicano esté adscrito a los servicios de atención.

En este trabajo se emplearon distancias lineales horizontales, lo cual ocasiona una subestimación de la 


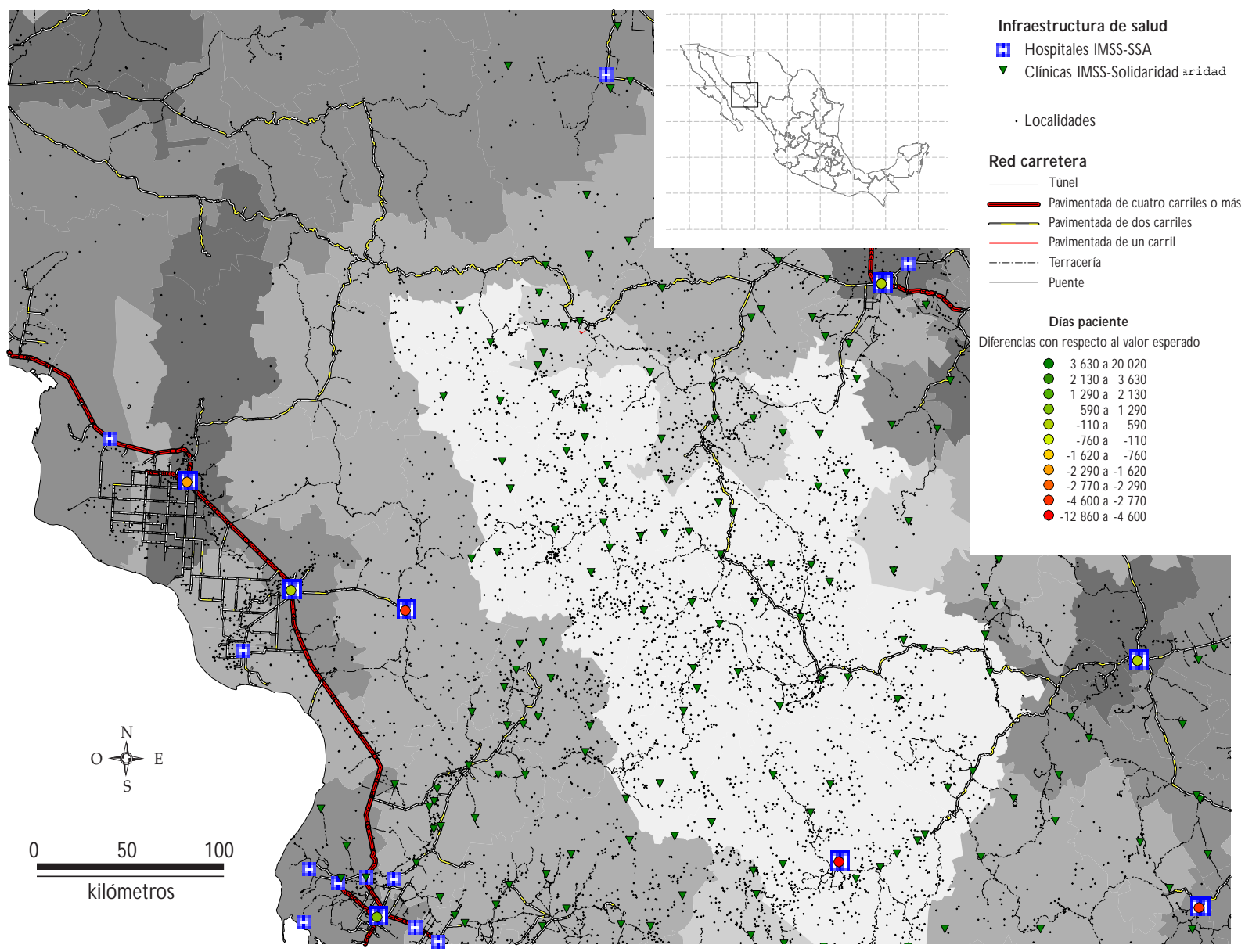

Figura 8. Distribución espacial de los residuales del modelo. Los residuales del modelo fueron geoREFERENCIADOS PARA INVESTIGAR LA DISTRIBUCIÓN ESPACIAL DE HOSPITALES GENERALES QUE OPERAN CON DIFERENCIAS EXTREMAS CON RESPECTO A LA MEDIA CALCULADA POR EL MODELO ESTADíSTICO. EN ESTA FIGURA SE PUEDE APRECIAR QUE VARIOS HOSPITALES CON BAJOS ÍNDICES DE OCUPACIÓN SE ENCUENTRAN ALREDEDOR DE ZONAS DONDE LA POBLACIÓN CAReCE de servicios de Segundo Nivel de atención, pero no tienen medios de acceso para llegar a ellos (CARRETERAS)

distancia verdadera. Este primer análisis podría refinarse con la incorporación de otras capas de información como la distribución de vías de comunicación terrestres; con la incorporación de esta información sería posible conocer las distancias reales entre la población y los servicios de salud, identificar las vías de acceso más cortas y proponer alternativas para localidades remotas. ${ }^{\text {? }}$

Adicionalmente, el análisis de la oferta (infraestructura y servicios) y la demanda (población usuaria) podría incorporar información sobre el lugar de residencia de los pacientes (para definir el área "real" de captación hospitalaria), sobre el servicio recibido por tipo de padecimiento, y el porcentaje de ocupación. En este contexto, al mostrar las áreas de captación real de cada hospital se podrían identificar con mejor precisión las coberturas geográficas que brindan los servicios de salud. Un sistema integral como éste, podría facilitar la distribución de recursos por hospital, de acuerdo con la población a la que atienden y al porcentaje de 
ocupación de cada unidad, proporcionando datos cuantitativos para la elaboración de convenios interinstitucionales e interestatales.

La gran variabilidad que muestra la $\mathrm{TAOH}$ en los hospitales estudiados conduce a un replanteamiento sobre la necesidad de flexibilizar su administración y otorgarles una mayor autonomía. Con esto se podrían incrementar los niveles de ocupación, a partir de la eliminación de barreras de tipo organizacional, con lo que se podrían generar mecanismos para que los hospitales puedan ofertar sistemáticamente su capacidad excedente, por ejemplo, a través de convenios o acuerdos con otras instituciones y la venta de servicios a personas físicas o morales.

Los SIG podrían ser muy útiles para materializar la línea de acción del Programa Nacional de Salud 20012006 en relación con la promoción de la autonomía de gestión hospitalaria y la gestión por contrato. Además, se podrá contar con mecanismos para establecer parámetros de productividad para la evaluación del desempeño y de la calidad.

Los residuales, interpretados como la diferencia entre el número de días paciente observados y los esperados según el modelo, nos indican la existencia de hospitales que operan con tasas de ocupación mayores o menores a la esperada, de acuerdo con su tamaño, el número de médicos y la presencia de otras unidades. Esto permitió la evaluación de hospitales de acuerdo con un valor medio esperado de ocupación (TAOH), dadas las características de cada unidad. Por ejemplo, el hospital general de Acapulco (punto 95 en la figura $7 \mathrm{~b}$ ), presentó una $\mathrm{TAOH}$ muy superior a la esperada para un hospital de sus características, mientras que el hospital general de Tehuacán, Puebla (punto 209 en la figura $7 \mathrm{~b}$ ), presentó una $\mathrm{TAOH}$ muy por debajo de lo esperado (cuadro IV). Con la geo-referenciación de los residuales, pudimos observar la distribución geográfica de los hospitales que operan con grandes diferencias en la TAOH (figura 8), su relación geográfica con otras unidades de atención de segundo y tercer nivel, así como con la población.

Las diferencias entre la TAOH observada, respecto al número de camas (figura 6a) y la TAOH ajustada por el modelo (figura 6c), así como la reducción de la variabilidad pueden ser utilizados para la evaluación continua del desempeño de los hospitales e incorporar los resultados en la planeación estratégica del sistema. Los resultados de este modelo de evaluación permitirán encontrar los niveles óptimos de ocupación hospitalaria que garanticen también elevados niveles en la calidad de la atención. Los hospitales cuya $\mathrm{TAOH}$ esperada es extremadamente baja (figura 6c) son indicadores de infraestructura ociosa. Un análisis particular de este grupo permitiría identificar los factores condicionantes de esta situación.

El signo de los coeficientes, más que en su interpretación cuantitativa, nos da una idea del tipo de asociación que existe entre la $\mathrm{TAOH}$ y las variables incluidas en el modelo. La asociación positiva encontrada entre el número de médicos de la SSA y del IMSS, presentes en la localidad donde se hallaba la unidad en estudio, y la TAOH podría explicarse por la correlación que hay entre la ubicación de unidades de la SSA y el IMSS con el tamaño de la localidad y ésta a su vez con la TAOH. La relación negativa que se observa con respecto al índice de marginación puede ser un indicativo de que la población prefiere atenderse en localidades de menor marginación o de que la población en localidades marginadas demanda menos servicios. Los patrones estacionales en la demanda y utilización de los hospitales generales podrían ser evaluados calculando tasas de ocupación (TADP y TAOH) para periodos específicos de tiempo. De esta manera, la evaluación de la demanda en temporadas invernales o de calor podría ser comparada, más aún, las tasas de ocupación también podrían ser específicas de acuerdo con los diagnósticos relacionados con estas temporadas.

El modelo ajustado explica gran parte de la variabilidad espacial incorporando los efectos de la correlación espacial en el análisis por buffers. La utilización de este tipo de modelos en la evaluación del desempeño permitirá analizar los índices de utilización nacionales, regionales y locales. Este sistema integral geo-referenciado del SMS, desarrollado con información de 1998, puede ser actualizado, con un mínimo de esfuerzo, con datos de años subsecuentes (incorporando así las tendencias temporales) y ser utilizado continuamente en la evaluación y planeación estratégica del sector.

Este sistema, basado en la demanda poblacional y en la identificación de necesidades, así como en la oferta de servicios, facilitaría la coordinación interinstitucional en el sector y conduciría a un mejor aprovechamiento de los recursos disponibles y a la planeación de nuevas unidades de atención.

La planeación estratégica en el sector salud, así como la investigación científica en salud pública y sistemas de salud se verían beneficiadas con un sistema como el presentado, que proporcione oportunamente la información necesaria, con el nivel de resolución geográfica adecuado para su análisis témporo-espacial, en diferentes escalas administrativas, regionales y locales. 


\section{Referencias}

1. Richards TB, Corner CM, Rushton G, Brown CK, Fowler L. Geographic information systems and public health: Mapping the future. Public $\mathrm{Health}$ Rep 1999;114:359-373.

2. Star J, Estes J. Geographic Information Systems:An introduction. N ueva York: Prentice Hall, 1990

3. Secretaría de Salud. Programa N acional de Salud 2001-2006. México D.F.: Secretaría de Salud, 2001.
4. Cleveland W S. Robust locally weighted regression and smoothing scatter plots. J Am Stat Assoc 1979;74:829-836.

5. Chambers JM, Cleveland W S, Kleiner B, Tukey PA. G raphical Methods for Data Analysis. Belmont (CA):W adsworth, 1983.

6. McC ullagh P, N elder JA. Generalized linear models. Segunda edición. N uevaYork: C hapman \& Hall, 1989:193-236.

7. Rushton G. Methods to evaluate geographical access to health services. J Public Health Manag Pract 1999;5:93-100. 